\title{
Etnografía digital de los sitios web de 10 estados en México para erradicar la violencia en contra de las mujeres
}

Digital ethnography of 10 Mexican states' websites to eradicate violence against women

Digital ethnography of 10 Mexican states 'websites to eradicate violence against women

\author{
Olga RODRIGUEZ CRUZ \\ Universidad Autónoma de la Ciudad de México (México) \\ olga.rodriguez@uacm.edu.mx \\ Yolanda GUERRA MACÍAS \\ Universidad Autónoma de la Ciudad de México (México) \\ yolanda.guerra@uacm.edu.mx
}

Chasqui. Revista Latinoamericana de Comunicación

N. ${ }^{\circ}$ 148, diciembre 2021 - marzo 2022 (Sección Diálogo de saberes, pp. 259-278)

ISSN 1390-1079 / e-ISSN 1390-924X

Ecuador: CIESPAL

Recibido: 2O-o9-2021 / Aprobado: 12-11-2021 


\begin{abstract}
Resumen
El objetivo fue indagar qué tipo de información contienen las páginas digitales de 10 estados y 76 municipios de México para disminuir el delito de violencia de género. La perspectiva teórica es la comunicación participativa. Se acudió a la etnografía digital para el estudio de los espacios digitales oficiales, para el análisis se diseñaron en cuatro categorías de análisis. Dentro de los principales hallazgos que se obtuvieron fueron que no existe un plan estratégico integral desde la federación ni de los gobiernos locales para intervenir, tampoco hay una estrategia de comunicación participativa en los distintos órdenes de gobierno para erradicar la violencia.
\end{abstract}

Palabras clave: comunicación participativa; cambio social; políticas públicas y Estado.

\begin{abstract}
The objective was to find out what type of information the digital pages of 10 states and 76 municipalities in Mexico contain to reduce the crime of gender violence. The theoretical perspective is participatory communication. Digital ethnography was used to study the official digital spaces, and the analysis was designed in four categories of analysis. Among the main findings obtained were that there is no comprehensive strategic plan from the federation or local governments to intervene, nor is there a participatory communication strategy in the different levels of government to eradicate violence.
\end{abstract}

Key words: participatory communication; social change; public policies; State.

\title{
Resumo
}

O objetivo foi investigar que tipo de informação as páginas digitais de 10 estados e 76 municípios mexicanos contêm para reduzir o crime de violência de gênero. A perspectiva teórica é a comunicação participativa. A etnografia digital foi utilizada para estudar os espaços digitais oficiais, para a análise eles foram designados em quatro categorias de análise. Entre as principais constatações obtidas está a inexistência de um plano estratégico abrangente da federação ou dos governos locais para intervir, nem uma estratégia de comunicação participativa nos diferentes níveis de governo para erradicar a violência.

Palavras-chave: comunicação participativa; mudança social; políticas públicas e do Estado. 


\section{Introducción}

México ocupa el primer lugar en el mundo por feminicidios, violencia de género y maltrato infantil. Se observa que, al menos que desde 1993 (Damián \& Flores, 2018), se publicaban en la nota roja situaciones de violencia intrafamiliar, desapariciones o violaciones de mujeres, principalmente en el estado de Chihuahua, esta problemática no era parte de la agenda política ni mediática de ninguno de los gobiernos: federal, estatal o municipal por contener este tipo de situaciones.

De acuerdo con la literatura teórica y empírica la violencia contra la mujer deviene de una serie de experiencias que ha vivido en diferentes etapas de su vida en donde la han agredido de forma sucesiva, en algunos casos llegan a normalizarse con el tiempo (Flores-Garrido Natalia \& Barreto-Ávila, 2018; M. S. Frías, 2014; S. Frías \& Castro, 2011). Este fenómeno es parte de la socialización de la violencia y se da en distintos espacios como la familia, la escuela, el trabajo, la relación de pareja, en ministerios públicos, entre otros (Bejarano \& Arellano, 2014; Evangelista-García et al., 2016; Henry et al., 2020).

Existen varios factores relacionados con la violencia intrafamiliar, entre ellos se encuentra el estrato socioeconómico que aumenta a medida que la pobreza es más marcada; pasa de igual manera si hay mayor número de hijos, así como la estructura en donde se acrecienta el riesgo en familias en donde sólo uno de los padres es biológico o en el caso de los padres adoptivos en donde las familias secundarias (pueden ser los abuelos, los tíos, etc.) son los que ejercen violencia en los niños o jóvenes. Asimismo, los factores contextuales como el desempleo, padres con problemas de drogadicción propician la violencia. Aunado a esto, el género de los hijos en donde los hombres adolescentes son maltratados físicamente más que las mujeres, pero en el caso de los niños, sufren de igual manera la violencia. Se ha de destacar que estudios de género en el ámbito de la desigualdad, muestran diversos aspectos de discriminación como los socioeconómicos, educativos y de etnia (Solís-Beltrán et al., 2018).

En el año 2010, la Secretaría de Gobernación emitió la primera Alerta de Violencia de Género (AVG) para el estado de Guanajuato (Damián \& Flores, 2018); sin embargo, todavía en la segunda década del siglo XXI, no todos los estados de la república mexicana han aceptado la AVG. En el año 2020, según cifras del Secretariado Ejecutivo del Sistema Nacional de Seguridad Pública (SESNSP) fueron asesinadas 6,861 mujeres, es decir, en promedio 18 féminas diariamente (Secretaría de Seguridad y Protección Ciudadana, 2020).

Es de resaltar, que, a pesar de los esfuerzos del movimiento feminista, académicas, cátedras en las universidades sobre la violencia de género, documentales, películas, entre otros medios que buscan expresar su preocupación e insatisfacción de la acción gubernamental por no atender este tipo de problemas sociales, que en lugar de ir disminuyendo la violencia contra las mujeres en todas sus manifestaciones ha ido en aumento. 
Actualmente hay algunos avances en materia de legislación para sancionar conductas que tienen que ver con la difusión, distribución, reproducción o comercialización de material audiovisual con contenido sexual no autorizado como es la Ley Olimpia (Gobierno de México, 2021), la cual ha sido aprobada por 29 estados de la república mexicana, las entidades que hacen falta son: Morelos, Nayarit y Tabasco. A diferencia de la Ley citada, la Ley Ingrid (Orden Jurídico, 2021) ha sido admitida únicamente en cuatro estados y con iniciativa de ley en tres más. Ésta busca tipificar las conductas, que al igual que la Ley Olimpia, tienen las mismas formas de dar a conocer un contenido; pero éste es relacionado con el delito de exhibir cadáveres o algunas partes del cuerpo, así como lesiones, estados de salud, detalles del fallecimiento, información reservada, hallazgos, entre otros elementos que buscan promover el morbo y obstaculizar una investigación. De manera particular, se menciona a los servidores públicos como sujetos de sanción, ya que son quienes han llegado a filtrar evidencia. En el caso de los estados en donde no se ha logrado ni siquiera la iniciativa de ley, se debe a argumentos por parte de los reporteros que apelan a la libertad de expresión.

Lo anterior es de preocupar, porque la literatura teórica nos habla que uno de los principales fines del Estado es garantizar seguridad a sus ciudadanos (Ferrater, 2001); si no cumple con éste habría que reconsiderar no solamente su funcionamiento, sino su estructura, es decir todo su sistema que puede llegar o es pernicioso para una sociedad, al no ejercer la justicia y la protección de las víctimas (Rawls, 2002).

Aunado a esto, los estudios han mostrado sobre la participación que tienen los medios de comunicación (Ramsoomar et al., 2021), en especial, las redes virtuales sociales en donde el uso de los medios digitales ha sido crucial para dar a conocer y posicionar al movimiento feminista; además de presentar y solicitar la cooperación de los usuarios para compartir información sobre niñas, adolescentes y mujeres desaparecidas; así como exhibir imágenes de agresores de mujeres para ser localizados. En este sentido, ha sido el movimiento feminista y la sociedad civil quienes han encarado este tipo de problemáticas. Es de subrayar, que el movimiento o los movimientos feministas, han sido las organizaciones más exitosas para la generación de capital social desde finales del siglo XIX hasta la actualidad.

De esta manera, la participación de la sociedad incide no solamente en visibilizar la violencia contra las féminas; sino también contribuye a generar capital social, el cual es detonante de la cooperación en una comunidad. No se puede hablar de comunicación participativa, si no hay condiciones para tejer vínculos de confianza, reciprocidad y solidaridad para el bien común (Bolton \& Ockenfels, 2000; Ostrom, 2000; Ostrom \& Ahn, 2003; Ostrom \& Walker, 2003).

El paradigma de la comunicación participativa se sitúa precisamente en la comunidad, es el punto de partida del proceso y que concibe a la comunicación de una manera horizontal para estimular la concientización de las personas 
como paso para una mejora sustancial de la calidad de vida (Barranquero, 2009, 2011, 2012; Barranquero \& Sáez, 2010; Beltrán, 2009; Díaz, 2004; Gumucio Dagron, 2004; Kaplún, 1998).

En este sentido, es importante considerar que el papel que tienen las redes sociales en el movimiento feminista, que si bien se habla de que se ha abierto la posibilidad de una comunicación horizontal en donde la población participa en las formas en que los grupos activistas generan sus protestas, lo anterior no es suficiente para combatir el problema (Segura-Mariño \& García-Umaña, 2019) es necesario una comunicación participativa desde las comunidades que se han visto afectadas por comportamientos violentos en contra de las mujeres.

Por ello, este artículo está destinado a indagar qué tipo de información contienen las páginas electrónicas de 10 estados y 76 municipios de México para disminuir el delito de violencia de género, con el fin de transformar entornos sociales para que las mujeres vivan tranquilas, seguras y tengan oportunidades de desarrollo en todas sus manifestaciones, en este sentido las políticas públicas tendrían que buscar intervenir en aspectos básicos como son: prevenir, atender, sancionar y erradicar cualquier violencia ejercida contra las féminas.

\section{Método}

La técnica de investigación empleada fue cualitativa, en específico se utilizó la etnografía virtual (Hine, 2004; Domínguez, 2007) la cual permitió visualizar, explorar y analizar de manera general 190 páginas electrónicas oficiales del gobierno de México (federación, estados y municipios). Se acudió a este tipo de método porque posibilitó indagar las características principales que tenían estos sitios web en cuanto a contenidos para erradicar la violencia en contra de las féminas.

Esimportante resaltar quela etnografía virtual no busca estudiar únicamente a sujetos o grupos en interacción en el ciberespacio (Domínguez, 2007), sus alcances son amplios, en este caso, es examinar las páginas digitales oficiales, pues se toma en cuenta que esta técnica parte de la idea, de que la tecnología ha transformado los espacios sociales, desde el momento en que presenta otras formas de aprehensión de la realidad, así como nuevos formas de comunicación y de reelaboraciones simbólicas (Ruiz y Aguirre, 2015).

Esta investigación se centró en la revisión de las páginas electrónicas en los 10 estados de la República mexicana que presentan el mayor número de feminicidios con el propósito de observar y responder a la pregunta ¿qué plan estratégico están desarrollando para prevenir, atender, sancionar y erradicar la violencia de género?

Los estados que se examinaron fueron: Estado de México, Ciudad de México, Nuevo León, Jalisco, Veracruz, Chihuahua, Coahuila, Puebla, Sinaloa, Sonora. Por ejemplo, en el mapa se atisba el lugar territorial de los estados enunciados, la $\mathrm{M}$ se refiere al número de municipios con los que cuenta cada entidad 
administrativa y después de la diagonal está el número de alcaldías en donde se da el fenómeno social y la $\mathrm{F}$ hace referencia al número de feminicidios en esas localidades.

Figura 1. Mapa República Mexicana

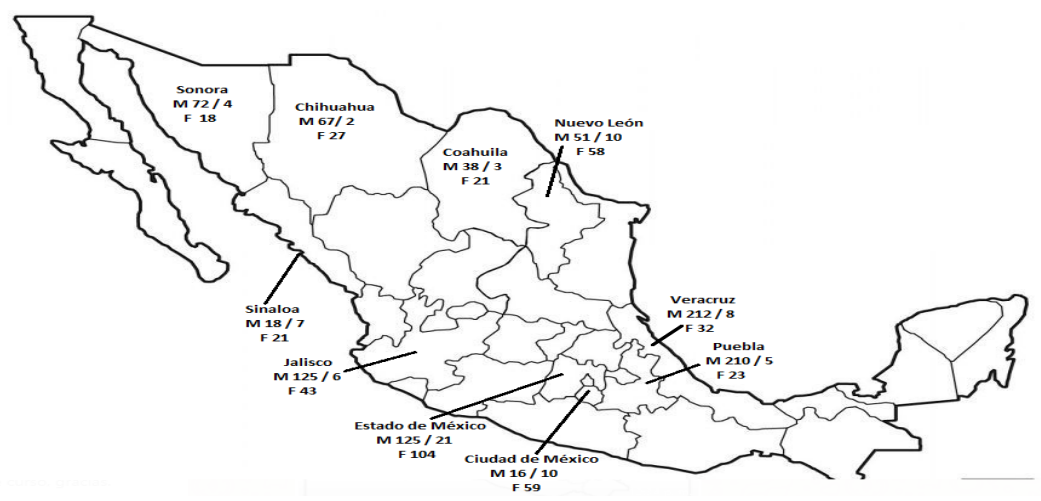

\section{Corpus de estudio}

México está constituido por 32 estados, en los cuales hay 2,446 Municipios. Para delimitar el corpus de estudio se decidió únicamente tomar como referencia los estados que presentaban el mayor índice de feminicidios. Por ello, se revisaron 190 páginas electrónicas oficiales de la federación como Inmujeres, Secretaría de Seguridad y Protección Ciudadana, además de 10 estados, 76 municipios y 78 espacios web sobre alerta de género y páginas relacionadas con este fenómeno.

Tabla 1. Control de datos por Estado y municipios

\begin{tabular}{|l|l|c|c|c|c|}
\hline \# Feminicidios & Estado & \# Municipio & $\begin{array}{l}\text { P. E. Vinculadas } \\
\text { a municipios }\end{array}$ & $\begin{array}{l}\text { Otras páginas } \\
\text { Alerta género }\end{array}$ & Total \\
\hline & Federación & & & & 2 \\
\hline 104 & Estado de México & 21 & 5 & 33 & 60 \\
\hline 59 & Ciudad de México & 10 & 5 & 10 & 26 \\
\hline 58 & Nuevo León & 10 & 1 & 4 & 16 \\
\hline 43 & Jalisco & 6 & 0 & 4 & 17 \\
\hline 32 & Veracruz & 8 & 3 & 9 & 21 \\
\hline 27 & Chihuahua & 2 & 1 & 6 & 10 \\
\hline 23 & Puebla & 5 & 1 & 3 & 10 \\
\hline 21 & Coahuila & 3 & 0 & 2 & 6 \\
\hline 21 & Sinaloa & 7 & 0 & 3 & 11 \\
\hline 18 & Sonora & 4 & 2 & 4 & 11 \\
\hline Total 406 & 11 & 76 & 18 & 78 & 190 \\
\hline
\end{tabular}

Elaboración propia. 


\section{Categorías de estudio}

Los indicadores para analizar fueron los siguientes:

- Primer indicador: La información sobre los programas para la prevención de la violencia es visible en los portales de cada uno de los 10 estados.

- Segundo indicador: La accesibilidad a la información es inmediata sobre qué hacer si las mujeres viven violencia en los portales electrónicos en los 76 municipios, que se reportan en esos 10 estados.

- Tercer indicador: Tipo de programas sociales para disminuir la violencia contra la mujer por estado y municipio: prevención, atención, sanción y erradicación.

- Cuarto indicador: Evaluación de los programas.

\section{Estrategia de búsqueda de la información}

Para la búsqueda de la información se hizo una revisión en el proyecto "Alerta de género", el cual muestra los estados que se han incorporado, además de que proporciona datos sobre las acciones que se han tomado y los estados con mayor índice de violencia. Aunado a eso se tuvo que hacer una exploración más detallada en cada uno de los estados y municipios para conocer las acciones y cuáles pertenecen a una estrategia de comunicación integral.

\section{Resultados}

Primer indicador: en la indagación se pudo constatar que solamente son tres de los 10 estados de la república mexicana, Veracruz, Chihuahua y Sonora, que en su portal electrónico hacen mención sobre la violencia en contra de la mujer. Veracruz, abiertamente expone que hay una Alerta de Violencia de Género (AVG), en su sitio web se observa que hay un Grupo Interinstitucional y Multidisciplinario (GIM) que fue constituido para dar seguimiento a la Alerta. No señala qué es la AVG, enuncia seis tipos de violencias: sexual, patrimonial, económica, psicológica, física y obstétrica (Gobierno del estado de Veracruz, 2021). El segundo estado es Chihuahua que únicamente muestra la violencia laboral, explica las fases de este fenómeno social, incluye un violentómetro laboral, proporciona teléfonos de la Procuraduría de la Defensa del Trabajo de seis de sus municipios, además de un chat en línea (Gobierno del estado de Chihuahua, 2021).

El último estado es Sonora que presenta una cintilla en su página web, en el que enuncia: Sitios de atención para alertas de la violencia de género en Sonora y ubica a las diversas instancias del estado y solamente una institución federal como es la Comisión Nacional para Prevenir y Erradicar la Violencia contra las Mujeres (Conavim) (Gobierno de Sonora, 2021). 
Segundo indicador: se identifica al Estado de México como el territorio que tiene el mayor número de municipios, 21, en el que se presenta el problema del feminicidio, de éstos solamente cinco ayuntamientos: Chalco, Nezahualcóyotl, Toluca, Teoloyucan, Ixtlahuaca, son los que incorporan información sobre el tema de violencia de género. Los tres primeros cuentan en su portal web con información y orientación sobre este tema, además, Chalco tiene una aplicación que se llama: Reacción mujer (Reacción Mujer Chalco, 2021). Teoloyucan solamente ofrece una aplicación y la nombra: Mujer segura. En el caso de Ixtlahuaca presenta un cártel y es en la liga de Desarrollo Integral de las Familias en el que se promueve la salud mental, en ningún momento se aborda el tema de la violencia. Estos municipios no son los que tienen el mayor número de casos registrados de feminicidio (Gobierno del estado de México, 2021).

La Ciudad de México es el segundo estado que presenta este tipo de violencia social en 10 de sus Alcaldías. Es de resaltar que Iztapalapa (Iztapalapa, 2021) es la jurisdicción que tiene el mayor problema, sin embargo, en su página electrónica no se atisba ninguna medida para erradicarla. Las administraciones que exhiben información son: Cuauhtémoc (Alcaldía Cuautémoc, 2021), Tlalpan (Alcaldía de Tlalpan, 2021), Gustavo A. Madero (Alcaldía Gustavo A. Madero, 2021), Venustiano Carranza (Alcaldía Venustiano Carranza, 2021), Miguel Hidalgo (Alcaldía Miguel Hidalgo, 2021), y Xochimilco (Alcaldía Xochimilco, 2021). Tanto los gobiernos locales de Cuauhtémoc y Miguel Hidalgo son los que ofrecen más datos y orientación sobre el tema, no pasa lo mismo con las demás alcaldías en donde se muestran banners fijos, que no aportan información para disminuir la violencia en contra de las mujeres. Es de resaltar que en la página electrónica de la Secretaría de las Mujeres del gobierno de la Ciudad de México sí contempla una serie de servicios de atención a las féminas que viven en este tipo de situación. En el ámbito de la prevención ofrece el programa Sendero seguro, en donde busca iluminar y habilitar espacios para que las mujeres caminen tranquilas. Asimismo, ha incorporado otros proyectos, por ejemplo: Viaja segura, Línea Mujeres, Abogadas de las mujeres en agencias de los ministerios públicos, entre otras tácticas (Gobierno de la Ciudad de México, 2021).

Veracruz ocupa la quinta posición. De sus ocho municipios registrados con este problema de violencia de género, solamente dos de éstos contemplan en sus páginas electrónicas este tipo de problema social, como son: Papantla (Red de mujeres por la Paz) (Papantla, 2021a, 2021b) y Coatzacoalcos (Instituto Municipal de la mujer) (Coatzacoalcos, 2021), este último aporta un número de WhatsApp para apoyo jurídico y psicológico y una encuesta para ser respondida por las mujeres; pero no proporciona suficiente información sobre el tema de la violencia de género.

En Puebla, de sus cinco municipios que cuentan con AVG, solamente es San Martín Texmelucan el que muestra en su portal web, información de un número telefónico de WhatsApp para que las mujeres que sufran algún tipo de violencia 
se comuniquen por medio de esa aplicación, a la cual llaman: Línea violeta (San Martín Texmelucan, 2021).

De los cuatro municipios de Sonora, únicamente Hermosillo presenta en su portal una encuesta que titula Pacto social por un Sonora Libre de la Violencia Política contra las Mujeres. Sorprende observar que la información está ubicada en el portal del Instituto Electoral del Estado (IEE Sonora, 2020) y aunque en su protocolo reconocen las diferentes manifestaciones de violencia, en general se encaminan solamente a la política.

Por otro lado, es de subrayar que Nuevo León ocupa el tercer lugar con el delito de feminicidio, sin embargo, de 10 ayuntamientos, el único municipio que tiene información es el de Guadalupe, en éste se vincula al Instituto Municipal de la Mujer (Municipio de Guadalupe, 2021).

Por su parte Jalisco ocupa el cuarto lugar en feminicidios, se atisba que no hay ni en su portal electrónico ni de sus municipios información sobre el problema. Los municipios que aparecen registrados son los siguientes ayuntamientos: Zapopan, Guadalajara, Tlajomulco de Zúñiga, San Pedro Tlaquepaque, Tonalá, El Salto. Lo mismo pasa con el estado de Sinaloa: Culiacán, Ahome, Mazatlán. El estado de Coahuila: Torreón, Acuña, Piedra Negras, Saltillo, Monclova, Sabinas y San Pedro.

El municipio que registra con mayor frecuencia el feminicidio es Ciudad Juárez, Chihuahua y no hay en su portal ninguna información para prevenir, atender, sancionar y erradicar la violencia de género (Secretaría de Seguridad y Protección Ciudadana, 2021).

Tercer indicador: en cuanto al tema de la intervención, se pudo ver que no todos los estados y municipios enunciados contemplan la prevención, atención, sanción y erradicación de la violencia en contra de la mujer. Esta información no está incluida dentro de los portales electrónicos mencionados; la obtención de datos fue posible por medio de otro tipo de búsqueda como fue la Alerta de Violencia de Género.

En el ámbito de la prevención, los municipios del estado de México que aparecen en una de las páginas de esa entidad son 11 de un total de 21 que se encuentran con la AVG: Chalco, Chimalhuacán, Cuautitlán Izcalli, Ecatepec, Ixtapaluca, Naucalpan de Juárez, Nezahualcóyotl, Tlalnepantla de Baz, Toluca, Tultitlán y Valle de Chalco (Gobierno del estado de México, 2021). En este último no hay ninguna información. En cuanto a los demás entre las formas de prevención se aprecia en la enunciación que una de las intenciones es el rescate de los espacios públicos, en algunos casos mencionan los lugares a rehabilitar, no señalan a qué se refieren con el término, en donde la mayoría coincide es con la instalación de alumbrado público.

Otros pocos, buscan concientizar a niños, adolescentes y adultos en los diferentes niveles de la educación formal, por medio de conferencias y obras de teatro. No exponen un plan de trabajo, ni los temas de este tipo de tácticas. 
Solamente tres ayuntamientos son los que consideran un trabajo con la comunidad, en los que destaca Tultitlán, en donde se manifiesta la construcción de redes juveniles y una red de mujeres contra la violencia. No hay más información sobre la organización, ni un plan de trabajo. En Ixtapaluca y Nezahualcóyotl también enuncian la importancia de este tipo de trabajo colaborativo en específico con mujeres. En ninguno de los municipios del Estado de México se especifica cómo se desarrollaron estas tácticas de prevención o si se cancelaron durante el periodo de confinamiento por la epidemia de SARS-CoV-2.

En el rubro de la atención se consideran tres aspectos: el primero, es el apoyo psicológico, jurídico y el autoempleo de las mujeres violentadas. El segundo, es la capacitación de servidores públicos con una perspectiva de género. El tercero, incluido en pocos ayuntamientos es la reeducación a hombres agresores, no se abordan mayores detalles sobre este tipo de trabajo de acompañamiento. La única administración que menciona a los Refugios temporales es Ecatepec.

En la parte de la sanción, en ninguno de los ayuntamientos del Estado de México se presenta un marco legal, que busque inhibir los comportamientos de los hombres violentos. Asimismo, tampoco se observa un plan estratégico para erradicar la violencia en contra de las mujeres.

En cuanto a la capital del país, Ciudad de México, en el terreno de la prevención implementó el programa de Sendero seguro, en donde habilitó con alumbrado público, cámaras de vigilancia y botón de emergencias (Gobierno de la Ciudad de México, 2021). En el año 2021 implementó un programa que está vinculado con la del gobierno federal nombrado como: Mujeres Constructoras de Paz (Inmujeres, 2021), en los que involucró a 232 féminas, quienes visitan 170 colonias en los que se dan el mayor número de violencia contra la mujer. La información no especifica ni Alcaldías ni colonias (Redacción kal, 2021).

Los aportes más importantes están orientados a la intervención en el marco legal, por ejemplo: en el registro de Bancos de ADN de los agresores, Registro público de los violentadores, Ley para que los hombres agresivos abandonen el hogar y no sea la mujer y los hijos quienes huyan. Este tipo de medidas se dan porque se detectan altos niveles de corrupción en el sistema de justicia penal; las violaciones al debido proceso y la ausencia de atención desde la perspectiva de género en todas las etapas que conllevan los procedimientos penales.

En el caso de Nuevo León, es interesante ver que dentro de las acciones que tiene para prevenir son en realidad medidas de atención en el sentido de capacitar únicamente a la policía para apoyar a las mujeres que son violentadas. Además de dar difusión de los derechos humanos con los que cuentan las féminas y la creación de un sistema de información. No se detalla más sobre este tipo de proyectos. Se aborda el tema de la impartición de justicia con perspectiva de género y sanción a los atacantes, pero no especifica más. Sobre las tácticas mediáticas únicamente desarrolla un mensaje de "Cero Tolerancia a la Violencia contra Mujeres y niñas” que rija la conducta de la función pública, 
pero no se observa en ninguna de sus propuestas de comunicación (Nuevo León, 2021).

En el estado de Jalisco, solamente hay en el caso de prevención la Instalación del Grupo Interinstitucional y Multidisciplinario (GIM) de la Alerta de Violencia de Género contra las Mujeres (AVGM) y el trabajo colaborativo con los municipios, de los cuales no se conocen sus planes estratégicos.

En cuanto a la atención se equiparon sedes del Centro de Justicia para las Mujeres en Guadalajara y Puerto Vallarta. Capacitación del personal que ayuda a las víctimas, entre ellas a la policía y el seguimiento policial a las denuncias de violencias de género para generar una red estatal de información y dar seguimiento a las áreas que dan órdenes y medidas de protección a mujeres, adolescentes y niñas. Señala la creación de un Banco Estatal de Casos e Información de Violencia contra las Mujeres en Jalisco (Baedavim), que proporciona información pública sobre el problema de la violencia de género (Gobierno del estado de Jalisco, 2021).

El estado de Veracruz busca la prevención por medio de tácticas educativas para modificar imaginarios culturales. Dice que pretende sensibilizar, capacitar y profesionalizar en Derechos Humanos de las Mujeres, en donde fortalecerá las unidades de género, pero no señala los públicos objetivo ni cómo lo logrará. Al igual que Nuevo León promoverá Cero Tolerancia de violencia contra mujeres, niñas y adolescentes en sus mensajes.

En la parte de atención, de manera similar a la Ciudad de México y Jalisco, Veracruz también incorpora un Banco Estatal de Datos e Información sobre Casos de Violencia contra las Mujeres (Banesvim) sin aportar más pormenores. Sobre la impartición de justicia dice que garantizará la reparación integral y simbólica del daño en casos de homicidios de mujeres y feminicidios. En lo que se refiere a los servidores públicos manifiesta que los supervisará y sancionará (Estado de Veracruz, 2016, 2021).

Chihuahua, de acuerdo con Conavim, recibió apoyo económico por parte de la ONU y la Unión Europea para tal fin; sin embargo, no hay información en su portal electrónico de ningún plan para disminuir este fenómeno social. En el sitio web del Instituto Chihuahuense de las Mujeres, hay un programa diseñado con objetivos que no dejan de ser de buenas intenciones, en el cual no se aborda el procedimiento para cumplirlos (Instituto Chihuahuense de las mujeres, 2017).

Puebla, de manera similar a Chihuahua tiene un plan de acción que contiene 45 medidas con sus objetivos y quiénes se encargarán de implementarlos; sin embargo, no dice ni cómo ni cuándo. Por su parte, la Comisión de Derechos Humanos (CDH) de este estado, instituyó el programa Mujer Segura en Casa, para otorgar apoyo psicológico a las féminas y motivarlas para que presenten su denuncia ante la Fiscalía General del Estado. A diferencia del gobierno del estado, la CDH de esta entidad ha solicitado medidas cautelares para la protección de las víctimas (Comisión de Derechos Humanos del estado de Puebla, 2017). 
Coahuila presenta una situación parecida a la de Chihuahua y Puebla, hay una serie de objetivos que no contemplan un plan de trabajo, son meramente enunciaciones (Estado de Coahuila, 2021). Lo único que se efectuó por parte del gobernador en marzo, en el Día de la Mujer, fue la entrega de "8o dispositivos de localización de agresores de mujeres”_(Instituto Coahuilense de las Mujeres, 2021).

En el caso de Sonora, es interesante observar que, en la Ley de acceso de las mujeres a una vida libre de violencia, se toma en cuenta el problema de la trata de personas (Gobierno de Sonora, 2021). Sin embargo, sucede lo mismo que en los otros estados, en donde solamente se enuncia, pero no se manifiesta cómo desarrollar ese plan estratégico. En Sinaloa el caso de buenas intenciones está señalado en la declaratoria de Alerta de Violencia de Género (AVG) (Estado de Sinaloa, 2017).

Es de destacar que el Instituto Nacional de las Mujeres (Inmujeres) que es parte del gobierno federal, en junio del año pasado dio a conocer su proyecto de Mujeres Constructoras de Paz (Mucpaz) que tiene como objetivo generar redes de mujeres en las localidades para trabajar en pro de la comunidad, en donde se resuelvan conflictos y sean parte de la transformación de la violencia en contra de éstas (Inmujeres, 2021).

Cuarto indicador: en ninguno de estos 10 estados y los 76 municipios se observó una evaluación a los programas que tienen en sus portales y que se localizaron en las páginas que contienen la información de Alerta de Violencia de Género. La Comisión Nacional de los Derechos Humanos presentó un diagnóstico 2019 en el que se evalúa el tema de la violencia contra las mujeres, da un informe general de la situación de toda la república mexicana; pero no hay una evaluación de los compromisos asumidos por los estados. Lo que sí señala es cómo estas entidades solicitan más recursos económicos, los cuales van principalmente al pago de nómina y no a desarrollar un plan estratégico para disminuir el problema (CNDH México, 2019).

\section{Conclusión}

De acuerdo con la investigación se observó que no hay interés por los diversos órdenes de gobierno por atender las Alertas de Violencia de Género. Se puede apreciar que no hay rendición de cuentas ni de responsabilidad hacia el Estado. Lo cual se traduce en una violencia institucional hacia las mujeres (Lagarde, 2018; Segato, 2017).

De la misma manera, se mira que no hay un trabajo comunicativo que apele a la concientización de la sociedad, pues el tema debe ser elaborado para los diferentes sectores de la población en donde se promueva la cultura de respeto hacia la mujer de cualquier edad, estrato social o nivel educativo. Dicha estrategia debe considerar una comunicación integral, pensada para promover el cambio social y respaldada por una serie de acciones en los gobiernos, que garantice 
el marco legal y la infraestructura para proteger a la mujer de cualquier tipo de agresión y a la par una educación permanente y sólida para la sociedad en general, en donde se promueva la cultura del respeto.

Por otro lado, es importante que la federación, los diferentes estados y municipios, dejen a un lado las diferencias políticas para concentrarse en un proyecto común que resuelva este grave problema, ya que serán directamente beneficiados con los resultados.

Finalmente, se debe tomar en cuenta que se está tratando con costumbres muy arraigadas en la sociedad mexicana que requieren un proyecto a largo plazo que incida en el cambio de conducta y que promueva de forma definitiva una cultura del respeto hacia la mujer.

\section{Referencias}

Alcaldía Cuauhtémoc. (2021). Equidad de género. Recuperado de https://alcaldiacuauhtemoc.mx/integra-genero/

Alcaldía de Tlalpan. (2021). Unidas y en comunidad. Recuperado de http://www.tlalpan. cdmx.gob.mx/unidas-y-en-comunidad/

Alcaldía Gustavo A. Madero. (2021). Salud mental GAM. Recuperado de http://www.gamadero.gob.mx/SaludMentalGAM/

Alcaldía Miguel Hidalgo. (2021). Red de prevención por la violencia de género. Recuperado de https://miguelhidalgo.cdmx.gob.mx/fortaseg/

Alcaldía Venustiano Carranza. (2021). Página oficial. Recuperado de https://www.vcarranza. cdmx.gob.mx/\#main-slider

Alcaldía Xochimilco. (2021). Página oficial. Recuperado de http://www.xochimilco.cdmx. gob.mx

Ayuntamiento de Netzahualcóyotl. (2021). Recuperado de Página oficial. http://www.neza. gob.mx

Barranquero, A. (2009). Latinoamérica en el paradigma participativo de la comunicación para el cambio (Universidad de Málaga, Ed.).

Barranquero, A. (2011). El espejismo de la comunicación para el cambio social. Radiografía de un concepto insostenible. Hacia una comunicación de cambio ecosocial. En Pontificia Universidad Javeriana (Ed.), Comunicación, desarrollo y cambio social: Interrelaciones entre comunicación, movimientos ciudadanos y medios, (pp. 81-100).

Barranquero, A. (2012). De la comunicación para el desarrollo a la justicia ecosocial y el buen vivir. CIC Cuadernos de Información y Comunicación, 17 (o). Recuperado de https://doi. org/10.5209/rev_CIYC.2012.v17.39258

Barranquero, A., \& Sáez, B. C. (2010). Comunicación alternativa y comunicación para el cambio social democrático: sujetos y objetos invisibles en la enseñanza de las teorías de la comunicación. Teorías y Métodos de Investigación En Comunicación Congreso Internacional AE-IC Málaga.

Bejarano, C. M., \& Arellano, G. M. del C. (2014). Violencia institucional contra las mujeres. Acta Sociológica, 97-120. Recuperado de https://www.sciencedirect.com/science/article/ pii/So186602814702385). 
Beltrán, S. L. R. (2009). Adiós a Aristóteles: la comunicación "horizontal." Punto Cero. Recuperado de http://www.scielo.org.bo/scielo.php? script=sci_arttext\&pi$\mathrm{d}=\mathrm{S} 1815$-02762007000200009

Bolton, G., \& Ockenfels, A. (2000). ERC: A Theory of Equity, Reciprocity, and Competition. American Economic Review 9o, pp.166-193.

CNDH México. (2019). Alerta de violencia de género contra las mujeres. Recuperado de https:// www.cndh.org.mx/sites/default/files/documentos/2019-11/Estudio-AVGM-2019.pdf

Coatzacoalcos. (2021). Instituto municipal de la mujer. Recuperado de https://www.coatzacoalcos.gob.mx/instituto-municipal-de-la-mujer/

Comisión de Derechos Humanos del estado de Puebla. (2017, February). Protocolo para la atención de presunta desaparición de mujeres y/o posible feminicidio. Recuperado de http://cdhpuebla.org.mx/images/cartillas/Protocolo_MujeresFeminicidio.pdf

Damián, B. A. L., \& Flores, J. A. (2018). Feminicidios y políticas públicas: declaratorias de alertas de violencia de género en México, 2015 - 2017. Perspectiva Geográfica, 23(2), 33-57. Recuperado de https://doi.org/10.19053/01233769.7287

Méndez, M. D. R. R., \& Aguilar, G. A. (2015). Etnografía virtual, un acercamiento al método y a sus aplicaciones. Estudios sobre las Culturas Contemporáneas. Vol. XXI. No. 41. Recuperado de https://www.redalyc.org/pdf/316/31639397004.pdf

Díaz, B. J. (2004). De la información agrícola a la comunicación para el cambio social. In Inta (Ed.), Comunicación, ruralidad y desarrollo: Mitos, paradigmas y dispositivos del cambio.

Domínguez F. D. (2007). Sobre la intención de la etnografía virtual. Revista Electrónica Teoría de la Educación. Educación y Cultura en la Sociedad de la Información. Vol. 8. $\mathrm{N}^{0} 1$. Mayo 2007. Recuperado de https://gredos.usal.es/bitstream/handle/10366/56552/ TE2007_V8N1_P42.pdf? sequence=1\&isAllowed=y

Estado de Coahuila. (2021). Sistema estatal de acceso de las mujeres a una vida libre de violencia. Recuperado de https://www.icmujeres.gob.mx/sistema-estatal/

Estado de Sinaloa. (2017, March 31). Declaratoria de alerta de violencia de género contra las mujeres. Recuperado de http://ismujeres.gob.mx/wp-content/uploads/2019/o2/ Declaratoria-de-AVGM-en-el-estado-de-Sinaloa.pdf

Estado de Veracruz. (2016). Declaratoria de alerta de violencia de género contra las mujeres estado de Veracruz. Recuperado de http://www.veracruz.gob.mx/wp-content/ uploads/2017/o6/Declaratoria_AVGM_Veracruz.pdf

Estado de Veracruz. (2021). Alerta de violencia de género. Recuperado de http://www.ivermujeres.gob.mx/avgm/

Evangelista-García, A. A., Tinoco-Ojanguren, R., \& Tuñón-Pablos, E. (2016). Violencia institucional hacia las mujeres en la región sur de México. LiminaR Estudios Sociales y Humanísticos.

Ferrater, M. (2001). Diccionario de filosofía. Barcelona: Ariel.

Flores-Garrido Natalia, \& Barreto-Ávila, M. (2018). Violencia en el noviazgo entre estudiantes de la Universidad Nacional Autónoma de México. Un análisis mixto. En Revista Iberoamericana de Educación Superior (Vol. 42, Issue 63, pp. 42-61). Universidad Nacional Autónoma de México. Recuperado de https://doi.org/10.22201/iisue.20072872e.2019.25.338

Frías, S. M. (2014). Ámbitos y formas de violencia contra mujeres y niñas: Evidencias a partir de las encuestas. Acta Sociológica, 65, pp. 11-36. Recuperado de http://www.un.org/

Frías, S., \& Castro, R. (2011). Socialización y violencia: desarrollo de un modelo de extensión de la violencia interpersonal a lo largo de la vida. Estudios Sociológicos, 29(86), pp. 497-550. Recuperado de https://www.jstor.org/stable/23043405 
Gobierno de Chalco. (2021). Página oficial. Recuperado de https://gobiernodechalco.gob.mx

Gobierno de la Ciudad de México. (2021). Secretaría de las mujeres. Recuperado de https:// www.semujeres.cdmx.gob.mx

Gobierno de México. (2021). Ley Olimpia. Recuperado de http://ordenjuridico.gob.mx/ violenciagenero/LEY\%20OLIMPIA.pdf

Gobierno de Sonora. (2021). Página oficial. Recuperado de https://www.sonora.gob.mx

Gobierno del estado de Chihuahua. (2021). Página oficial. Recuperado de http://www.chihuahua.gob.mx

Gobierno del estado de Jalisco. (2021). Alerta de violencia de género contra las mujeres. Recuperado de https://www.jalisco.gob.mx/es/gobierno/comunicados/alerta-de-violencia-de-genero-contra-las-mujeres

Gobierno del estado de México. (2021). Alerta de género. Recuperado de http://alertadegenero.edomex.gob.mx/municipios_alerta

Gobierno del estado de Veracruz. (2021). Página oficial. Recuperado de http://www.veracruz. gob.mx; http://www.veracruz.gob.mx/avgm/

Gumucio Dagron, A. (2004). El cuarto mosquetero, la comunicación para el cambio social, investigación y desarrollo: Vols. 12 (1), 23 (Universidad del Norte, Ed.). Recuperado de http://redalyc.uaemex.mx/pdf/268/26800101.pdf

Henry, N., Flynn, A., \& Powell, A. (2020). Technology-Facilitated Domestic and Sexual Violence: A Review. Violence Against Women, 26(15-16), pp. 1828-1854. Recuperado de https:// doi.org/10.1177/1077801219875821

IEE Sonora. (2020). Protocolo para la atención de la violencia política contra las mujeres en razón de género en Sonora. Recuperado de http://ieesonora.org.mx/protocoloVPMG

Inmujeres. (2021). Red de mujeres constructoras de paz. Recuperado de https://www.gob. $\mathrm{mx} /$ inmujeres/acciones-y-programas/red-de-mujeres-constructoras-de-paz-mucpaz

Instituto Chihuahuense de las mujeres. (2017). Programa institucional para garantizar el derecho de las mujeres a una vida libre de violencia. Recuperado de http://ceg.chihuahua. gob.mx/PED/EJE1/MedianoP/ICHMUJERES.PDF

Instituto Coahuilense de las Mujeres. (2021, March 12). Fortalecimiento de la prevención $y$ atención de la violencia contra las mujeres. Recuperado de https://coahuila.gob.mx/ agenda/evento/6050def86ofaco2bo58b4669

Ixtlahuaca. (2021). Página oficial. Recuperado de https://ixtlahuaca.gob.mx

Iztapalapa. (2021). Página oficial. Recuperado de http://www.iztapalapa.cdmx.gob.mx

Kaplún, M. (1998). Una pedagogía de la comunicación. Madrid: Ediciones de la Torre.

Lagarde, M. (2018). Género y feminismo: desarrollo humano y democracia. México-Argentina: Siglo XXI.

Municipio de Guadalupe (2021). Instituto municipal de la mujer de Guadalupe. Recuperado de http://inmujerguadalupe.gob.mx

Nuevo León. (2021). Alerta de violencia de género contra las mujeres. Recuperado de https:// www.nl.gob.mx/campanas/alerta-de-violencia-de-genero-contra-las-mujeres

Orden Jurídico. (2021). Ficha técnica Ley Ingrid. Recuperado de http://ordenjuridico.gob. $\mathrm{mx} /$ violenciagenero/LEY\%2OINGRID.pdf

Ostrom, E. (2000). El gobierno de los comunes. La evolución de las instituciones de acción colectiva (FCE, UNAM, \& CRIM, Eds.). Recuperado de https://www.crim.unam.mx/web/ sites/default/files/El\%2ogobierno\%20de\%2olos\%2obienes\%20comunes.pdf

Ostrom, E., \& Ahn, T. k. (2003). Una perspectiva del capital social desde las ciencias sociales: capital social y acción colectiva. Revista Mexicana de Sociología, pp. 155-233. Recuperado 
de http://www.scielo.org.mx/scielo.php?pid=So188-25032003000100005\&script=sci_abstract

Ostrom, E., \& Walker, J. (Eds.). (2003). Trust and Reciprocity: Interdisciplinary Lessons from Experimental Research. Nueva York: Russell Sage Foundation.

Papantla. (2021a). Página oficial. Recuperado de https://papantlaveracruz.com.mx

Papantla. (2021b). Red de mujeres por la paz. Recuperado de https://papantlaveracruz.com. $\mathrm{mx} /$ mujeres-por-la-paz/

Ramsoomar, L., Ladbury, R., \& Jewkes, R. (2021). Research uptake, lessons from a multi-country global programme: What Works to Prevent Violence against women and girls. Development in Practice, o(o), pp. 1-13. Recuperado de https://doi.org/10.108o/o 9614524.2021.1911952

Rawls, J. (2002). Teoría de la justicia social. México: Fondo de Cultura Económica.

Reacción Mujer Chalco. (2021). Prevención de violencia de género. Recuperado de https:// reaccionmujer.gobiernodechalco.gob.mx

Redacción kal. (2021, February 25). ¿Qué resultados ha dado la alerta de género en CDMX? Chilango. Recuperado de https://www.chilango.com/noticias/resultados-de-la-alerta-de-genero-cdmx/

San Martín Texmelucan. (2021). Página oficial. Recuperado de https://sanmartintexmelucan.gob.mx

Secretaría de Seguridad y Protección Ciudadana. (2021). Informe de actividades del Consejo Nacional de Seguridad Pública. Recuperado de https://www.gob.mx/cms/uploads/attachment/file/604121/4InformeActividadesXLVI.pdf

Secretaría del Trabajo y de Previsión Social. Gobierno de Chihuahua. (2021). Página oficial. Recuperado de https://www.stpschihuahua.com/cuentalo

Segato, R. (2017). La estructura de género y el mandato de violación. Caballero, Edith; de Santiago, Alejandra y González, Gabriela, Mujeres intelectuales: feminismo y liberación en América Latina y el Caribe. Buenos Aires: Clacso, pp. 299-332. Recuperado de https:// diwqtxts1xzle7.cloudfront.net/55026691/Antologia_Mujeres_Intelectuales-with-cover-page-v2.pdf? Expires $=1632180118 \&$ Signature $=$ PqJD9GqDFFH2-gLEi77V1R89IdRQjtVhNdqcDkgaxRJ3lVMeDpSECJyG-JhP2nTqy7Hvp2bgOv7eyyV6d2H44CMpLrtrDbaZaGvwquU7ngTGzxoEKoK7AECeA1XIFKzgg9rXcV7THg1kVQna4LkGii6rgiH8qCIYWtOf12T9DNbuvpASTjroEvmik71HF7hkEmvorhoxGbz4BmXwDbhY2TgXNCXQAtrMAcfaoG96gwJbIsR3YLdXJ-UDtiHvapimteZSJ4RSJTvhUECmW-esQhtZVqGiFoaQMEkaAV oz-aOHXFviRywPZy8xWBubB $\sim$ PTjmz $\sim$ qXPIıwR-MbEoKFoKw_\&Key-Pair-Id=APKAJLOHF5GGSLRBV4ZA\#page $=300$

Segura-Mariño, A.-G., \& García-Umaña, A. (2019). El arte digital y su relación con la violencia de género. Revista de Humanidades, 38, pp.65-84. Recuperado de https://dialnet.unirioja. es/servlet/articulo? codigo $=7139083$

Solís-Beltrán, G., Fernández-Ronquillo, M., Solís-Granda, L., \& Terán-Puente, C. (2018). Desigualdad de género en procesos educativos incidente en violencia hacia la mujer. PODIUM, (33), pp. 13-24. Recuperado de https://doi.org/10.31095/podium.201

Teoloyucan. (2021). Página oficial. Recuperado de https://www.teoloyucan.gob.mx

Toluca. (2021a). Instituto Municipal de la Mujer. Recuperado de https://www2.toluca.gob. $\mathrm{mx} /$ mujeres/

Toluca. (2021b). Página oficial. Recuperado de https://www2.toluca.gob.mx 
ETNOGRAFÍA DIGITAL DE LOS SITIOS WEB DE 10 ESTADOS EN MÉXICO... 
ETNOGRAFÍA DIGITAL DE LOS SITIOS WEB DE 10 ESTADOS EN MÉXICO... 
